\title{
Effects of large laser bandwidth on stimulated Raman scattering instability in underdense plasma
}

Yao Zhao, ${ }^{1}$ Lu-Le Yu, ${ }^{1}$ Jun Zheng, ${ }^{1}$ Su-Ming Weng, ${ }^{1}$ Chuang Ren,,${ }^{2,3}$ Chuan-Sheng Liu, ${ }^{4}$ and Zheng-Ming Sheng ${ }^{1,5, \text { a) }}$

${ }^{1)}$ Key Laboratory for Laser Plasmas (MoE) and Department of Physics and Astronomy,

IFSA Collaborative Innovation Center, Shanghai Jiao Tong University, Shanghai 200240,

China

${ }^{2)}$ Department of Mechanical Engineering and Laboratory for Laser Energetics, University of Rochester, Rochester,

New York 14627, USA

${ }^{3)}$ Department of Physics and Astronomy, University of Rochester, Rochester, New York 14627, USA

${ }^{4)}$ East-West Space Science Center, University of Maryland, College Park, Maryland 20742,

USA

${ }^{5)}$ SUPA, Department of Physics, University of Strathclyde, Glasgow G4 ONG, UK

(Dated: 29 April 2015)

The effects of laser bandwidth on stimulated Raman scattering (SRS) instability in underdense plasma are studied by particle-in-cell simulations. In the simulations, sinusoidal frequency modulation of the incident laser pulse is used. By changing the size of bandwidth, it is shown that the linear growth of SRS can be suppressed considerably, provided the laser bandwidth is much larger than the SRS linear growth rate. Simulations also show that by choosing the proper frequency modulation parameters or decreasing the linear growth rate of SRS, the inhibitory effects become more obvious. The plasma electron temperature tends to weaken the bandwidth effects especially when it is over a keV level. The laser bandwidth can only increase the time duration for linear growth but cannot diminish the instability completely.

\section{INTRODUCTION}

Stimulated Raman scattering (SRS) in plasma is a subject which has been widely investigated in the last decades ${ }^{1}$. It is related to a variety of applications, such as inertial confinement fusion ${ }^{2}$, high field nonlinear optics ${ }^{3}$, laser-plasma accelerators ${ }^{4}$ and new radiation source ${ }^{5}$. A key nonlinear phenomenon in SRS is the pump laser scattered by the electron density perturbation of a plasma wave, ${ }^{6}$ which is usually disadvantageous for inertial confinement fusion since it causes significant laser energy loss and can produce harmful hot electrons to preheat fusion targets ${ }^{7-10}$. In the past decades, several methods have been proposed to suppress the SRS, and most of them rely on the manipulation of laser pulses, such as temporal and spatial laser beam smoothing ${ }^{1,11-13}$.

In 1974, Thomson and Karush proposed to use finite laser bandwidths to suppress parametric instabilities ${ }^{12}$. Later, Obenschain et al. experimentally confirmed the suppression effects of bandwidth on instabilities. Due to the limitation of the laser technology, the bandwidth used in the experiment was $\triangle \omega=3 \times 10^{-3}$, where $\triangle \omega$ is normalized by the frequency of the incident laser $\omega_{0} .{ }^{14}$ In 1991, Guzdar et al. studied the bandwidth effects in inhomogeneous plasmas. ${ }^{15}$ In 2001, Evan et al. found that a linear frequency chirp with $12 \%$ bandwidth can eliminate Raman forward scattering for a plasma density that is $1 \%$ of the critical density. ${ }^{6}$ Besides the temporal modulations of the laser pulses, effects of spatial modulations on parametric instabilities have been also studied ${ }^{11}$.

a)zmsheng@sjtu.edu.cn or zhengming.sheng@strath.ac.uk
Recent developments on laser technology suggest that it is possible to generate laser pulses with broad bandwidths by using noncollinear optical parametric chirped-pulse amplification technology $y^{16,17}$. However, the bandwidth effects on SRS have not yet received sufficient attention so far in the context of inertial confined fusion. Also even though it is found that the laser bandwidth can reduce the linear growth rate of SRS, it is still not clear how the laser bandwidth would affect the nonlinear process of SRS. In this paper, we study all these bandwidth effects via particle-in-cell (PIC) simulations.

\section{THE COMPARISON BETWEEN PIC SIMULATIONS AND ANALYTICAL MODEL}

\section{A. The introduction of laser bandwidth model}

To study the effects of laser bandwidth on SRS, and compare the simulations with the analytical model, sinusoidal frequency modulation is used in our PIC simulations. The temporal part of the incident laser is as follows

$$
E_{z}=a_{0} \cos \left[2 \pi t+b \sin \left(2 \pi f_{m} t\right)\right]
$$

where $a_{0}$ is the amplitude of the laser vector potential, which is normalized by $m_{e} \omega_{0} c / e$, with $c$ the speed of light, $\omega_{0}$ the laser frequency, $e$ and $m_{e}$ the electron charge and mass, respectively. $t$ is normalized by the laser period $\tau=2 \pi / \omega_{0}$. The relation between the laser intensity $I_{0}$ and $a_{0}$ is $a_{0}^{2} \simeq$ $7.3 \times 10^{-19}[\lambda(\mu \mathrm{m})]^{2} I_{0}\left(\mathrm{~W} / \mathrm{cm}^{2}\right)$. Under the inertial confinement fusion conditions, $a_{0}$ should be limited to the range from 0.01 to 0.1 , so the corresponding intensity of the laser is much smaller than the relativistic threshold. The bandwidth of $E_{z}$ 


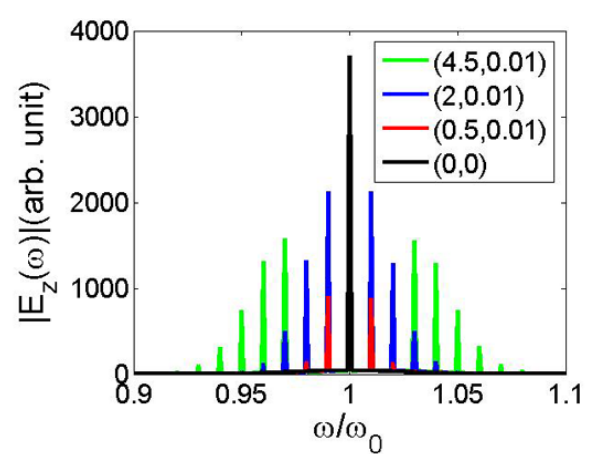

FIG. 1. Fourier transform of the incident lasers with finite bandwidth under different $\left(b, f_{m}\right)$ parameters.

can be approximated as $\triangle \omega=(2 b+1) f_{m}$. There are several peaks in the frequency spectrum, and the interval of each peak is $f_{m}$. When $\triangle \omega$ is a constant, too large $f_{m}$ leads to small number of peaks in spectrum, while $f_{m}$ is too smal1 , the discrete spectrum becomes approximately continuous. Figure 1 shows an example of the spectra of incident laser with finite bandwidth in the form of Eq. (1). The maximum bandwidth shown is $10 \%$, which corresponds to the parameter $\left(b, f_{m}\right)=(4.5,0.01)$, and the minimum bandwidth is 0 , i.e., $\left(b, f_{m}\right)=(0,0)$. The gap between two neighboring spikes is $\delta \omega=f_{m}=0.01$.

In the analytical model, the SRS linear growth rate is $\Gamma \sim$ $\Gamma_{0}^{2} / \triangle \omega$, provided that the bandwidth $\triangle \omega$ is much larger than the linear growth rate $\Gamma_{0}$ without bandwidth ${ }^{12}$. It indicates that the growth rate can be significantly reduced by using a large bandwidth. The maximum growth rate of stimulated Raman backscattering is $\Gamma_{0}=\sqrt{3}\left(\omega_{0} \omega_{p}^{2} a_{0}^{2} / 16\right)^{1 / 3} \cdot{ }^{18}$ For example, if taking the laser amplitude $a_{0} \sim 10^{-2}$, the plasma density is $n_{e} \sim 10^{-2}$, where $n_{e}$ is normalized by critical density $n_{c}=m_{e} \omega_{0}^{2} / 4 \pi e^{2}$, then the linear growth rate of the SRS is $\Gamma_{0} \simeq 0.007$. In this case, when the bandwidth $\triangle \omega \gtrsim 10^{-2}$, the suppression of the SRS growth will be obvious. To verify the analytical model, we set the bandwidth to be $2 \%, 5 \%$, and $10 \%$ in our simulations, and compare the SRS growth process with the case without bandwidth. Analytical theory is compliant only when $\Delta \omega \gg \Gamma_{0}$. However, in the real applications, this theory is valid only for the limited laser-plasma parameters. Therefore, PIC simulations are very necessary to study the suppression of the SRS by using laser pulses with finite bandwidths. In the following, our simulations show that the bandwidth suppression effects using different laser-plasma parameters. The larger bandwidth, the stronger suppression we can see. It is also found that when the growth rate closes to the bandwidth, the simulation results do not fit with the analytical model well.

\section{B. One-dimensional PIC simulations}

Numerical simulations using the PIC code $\mathrm{KLAP}^{19}$ have been formed both in one-dimensional (1D) and two- dimensional (2D) cases. We start with the 1D simulation. The length of the simulation box is $1260 \lambda$ with 100 cells per wavelength, where $\lambda$ is the incident laser wavelength in vacuum. We put 50 particles per cell. Left vacuum and right vacuum occupy $230 \lambda$ and $30 \lambda$, respectively, and the rest is occupied by plasma. In the front of the plasma, a $10 \lambda$ linear density ramp is added. The normalized homogeneous plasma density is $n_{e}=0.08$, and the temperature is $T_{e}=100 \mathrm{eV}$. The semiinfinite pump laser is s-polarized with the uniform amplitude $a_{0}=0.05$, with a $25 \lambda$ rising edge in the front. The ion mass is $m_{i}=1836 m_{e}$. The pump pulse duration used in ICF is typically about a few nanoseconds ${ }^{10}$, the saturation of SRS can be found at a few picoseconds. Therefore our simulation presented below is limited to $t=1500 \tau$.

The backward lights are diagnosed at $x=5 \lambda$, and the temporal evolution of the backscattering light is given in Fig. 2(a). It shows that there are very small differences between cases with 0 and $2 \%$ bandwidth. However, when $\triangle \omega=5 \%$, the amplitude of backscattering light grows slowly as compared to the case without bandwidth. This shows that the growth rate of SRS is reduced by using a 5\% bandwidth. It grows even slower when $\triangle \omega=10 \%$. These results are in good agreement with the theory, i.e., when the bandwidth is much larger than $\Gamma_{0}$, the SRS can be suppressed obviously, and the larger bandwidth, the stronger suppression effects are. The frequency spectra of the backscattering lights are shown in Fig. 2(b). One can see that the spectra contain two parts: one located at 0.72 is produced by the SRS, and the other located near 1.0 is produced by the stimulated Brillouin scattering (SBS). One may note that the spectrum is broadened when the bandwidth is added, which can be explained as $\omega_{n}=\omega_{0}-\omega_{p} \pm n f_{m}$, with $\omega_{p}$ the frequency of plasma wave and $n$ an integer. It is clearly seen that the intensity of the SBS spectrum is reduced when $\triangle \omega \geq 5 \%$, indicating that the bandwidth can suppress the SBS. ${ }^{12,20-22}$ Figure 2(c) describes the forward light spectra at $x=400 \lambda$. There are two peaks at $\omega=0.71 \omega_{0}$ and $\omega=1.25 \omega_{0}$ that correspond to the Stokes and anti-Stokes SRS forward scatteringn. Their intensities are reduced when the bandwidths become larger. We can also see a peak near $\omega=0.41 \omega_{0}$, which comes from the backscattering of simulated Raman backscattering ${ }^{23,24}$. It can be deduced from $\omega=\omega_{0}-2 \omega_{p}=0.43 \omega_{0}$. The slight frequency difference between theoretical value and simulation result can be attributed to the thermal frequency up-shift of the Bohm-Gross wave by a factor of $\sqrt{1+3 k^{2} v_{t h}^{2} / 2 \omega_{p}^{2}} \cdot{ }^{25,26}$ The corresponding wave vector is $k=k_{1}+\left|k_{2}\right|=0.98$ which is normalized by $\omega_{0} / c$, where $k_{1}$ is the wave vector of the backscattering light at the first time, $k_{2}$ is the wave vector of the backscattering light at the second time. This is shown later in Fig. 3(b).

To emphasize the bandwidth effects, we compare the SRS with $\triangle \omega=0$ and $\triangle \omega=10 \%$. To characterize the intensity of the SRS, we study the electron density $n_{e}$ and longitudinal electrostatic field $E_{x}$. Figures 3(a) and (c) show that at $t=600 \tau$, the amplitudes of density perturbation $n_{e}$ and longitudinal electric field $E_{x}$ are reduced in the early stage of laser propagation when $\triangle \omega=10 \%$, implying that the growth time has been delayed. Since the linear growth rate of the SRS 

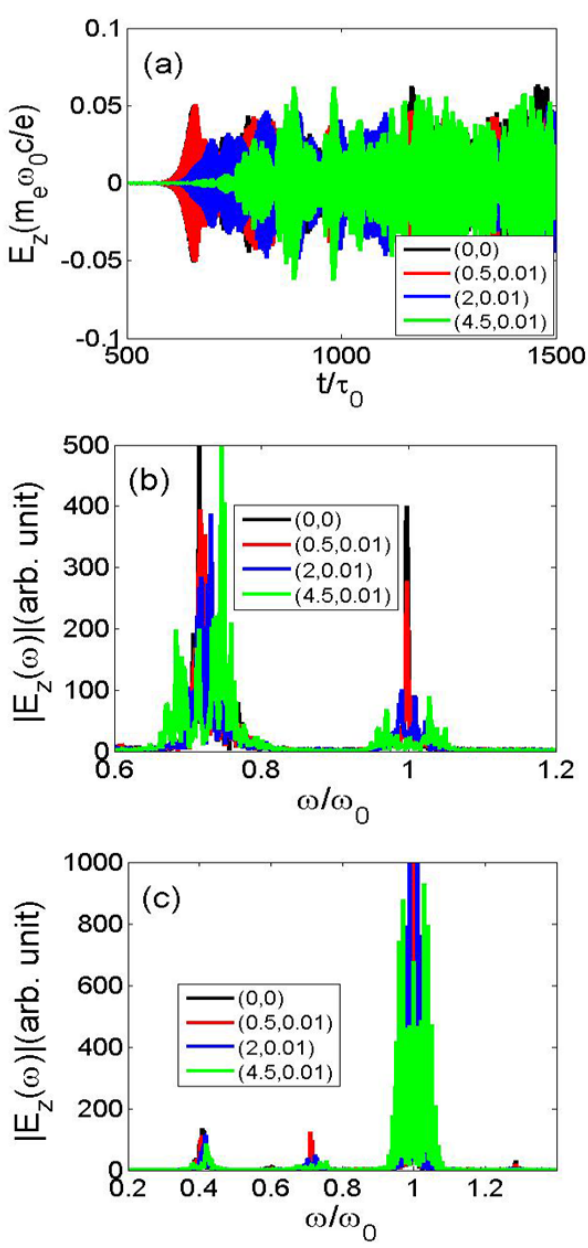

FIG. 2. (a) Temporal evolution of the backscattering light with finite bandwidth under different $\left(b, f_{m}\right)$ parameters at $x=5 \lambda$. (b) Fourier transform of the backscattering light in (a), which is taken in the time window from $500 \tau$ to $1500 \tau$. (c) Frequency spectra of forward scattering light at $x=400 \lambda$. The normalized amplitude of the incident laser is $a_{0}=0.05$ and the plasma density is $n_{e}=0.08$.

has been reduced, it can delay the developing time of rescattering process as mentioned above. As shown in Fig. 3(b), the wave vector of the rescattering light at $k=0.98$ is found only in the case without bandwidth, which is responsible for the spectrum peak near 0.41 as shown in Fig. 2(c). At later time, however, SRS in the case with a large bandwidth is found to grow to a level comparable to that without bandwidth as shown in Fig. 3(d) for the longitudinal electrostatic fields $E_{x}$ at $t=1200 \tau$. This indicates the suppression effects become weaker with the increase of the laser-plasma interaction time. The analytical model with finite bandwidth based on the three wave interactions indicates that bandwidth can suppress the SRS linear growth rate, however, the bandwidth is not a damping mechanism to determine the saturation level. When the laser-plasma interaction time becomes longer, SRS development not only depends upon the three-wave interaction, but also upon the wave-particle interaction. The SRS saturation level appears to be determined by the interaction between the
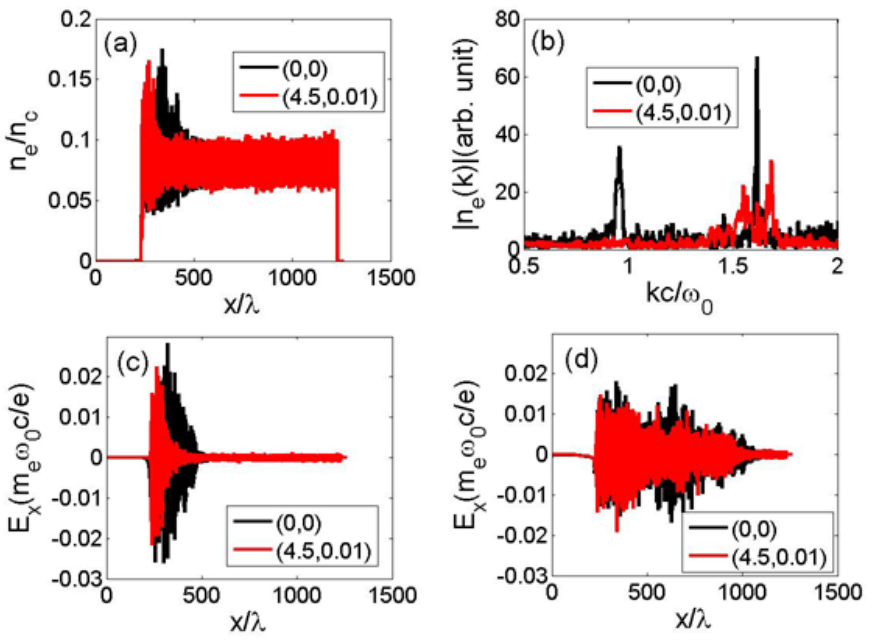

FIG. 3. Comparison of the electron density, longitudinal momentum, and longitudinal electric field with or without finite bandwidth. (a) Electron density at $t=600 \tau$. (b) Fourier transform of plot (a). (c) and (d) Longitudinal electric field at $t=600 \tau$ and $t=1200 \tau$, respectively. The normalized amplitude of the incident laser is $a_{0}=$ 0.05 and the plasma density is $n_{e}=0.08$.

electron plasma wave and electrons ${ }^{1,27-30}$, i.e., the damping of the electron plasma wave via electron trapping and subsequent heating determines its saturation amplitude. As shown in Fig. 3(d), the saturation levels of the longitudinal electrostatic fields for the two cases with or without finite laser bandwidth are similar. As shown later, in the nonlinear stage, electrons are heated to the similar level between the cases with or without frequency bandwidth.

Theoretical model predicted that the linear growth rate satisfies the function $\Gamma=\Gamma_{0} J_{0}(b),{ }^{12}$ where $\Gamma_{0}$ is the SRS linear growth rate without bandwidth, and $J_{0}$ is the first kind Bessel function with zero order. To compare the theory with simulation results, we set the parameters as $n_{e}=0.1, a_{0}=0.08$, $f_{m}=0.1$, and $b=0,0.2,0.4,0.6,0.8,1$ that corresponds to the bandwidths $\triangle \omega=0,14 \%, 18 \%, 22 \%, 26 \%, 30 \%$, respectively. Figure 4(a) shows the temporal evolution of the backscattering light. It is clearly seen that when the bandwidth becomes larger, the amplitude of the backscattering light grows more slowly. We use an exponential function $\left.E_{z}=R+r \exp ^{(} \Gamma t\right)$ to fit the envelope of the backscattering light, where $R$ and $r$ are the fitting parameters, and $t$ is the laser-plasma interaction time. From the fitting, we obtain the growth rate $\Gamma$, as plotted in Fig 4(b). It shows that the linear growth rate as a function of the modulation parameter $b$, which qualitatively agrees with the analytical model, but quantitatively they are different. From the simulation, we get

$$
\Gamma=\Gamma_{0} J_{0}(b)^{2.5} \text {. }
$$

In fact, the theory is applicable when $f_{m} \gg \Gamma_{0}$. However, under laser fusion conditions, the growth rate $\Gamma_{0}$ could be close to $f_{m}$. 

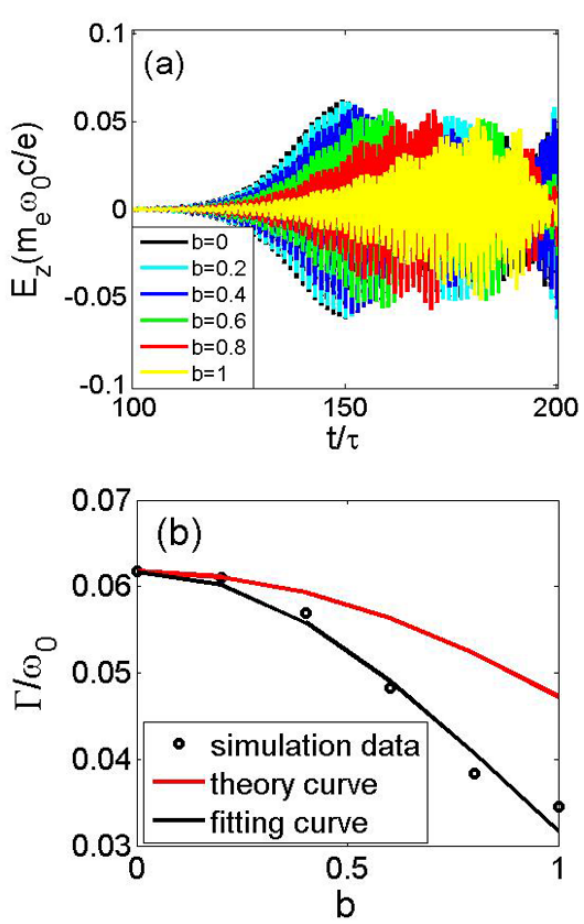

FIG. 4. Temporal evolution of the backscattering light and corresponding linear growth rate. (a) Temporal evolution of the backscattering light, with a constant $f_{m}$ and different $b$. Plasma density is $n_{e}=0.1$, laser parameters are $a_{0}=0.08, f_{m}=0.1$. The dot in (b) is the linear growth rate which is calculated from (a), the black solid line is the fitting curve of the simulation data, and the red solid line is the analytical model given in Ref. [12].

\section{THE INFLUENCE OF LASER AND PLASMA PARAMETERS ON SUPPRESSION EFFECTS FROM TWO-DIMENSIONAL PIC SIMULATIONS}

\section{A. Bandwidth effects}

There are some phenomena that cannot be observed by 1D PIC simulations, such as SRS side scattering. So in this section, we study the bandwidth effects in 2D PIC simulations, and compare the results with $1 \mathrm{D}$ cases. Meanwhile we will show the effects of the bandwidth on the SRS side scattering.

The length and width of our simulation box are $300 \lambda$ and $60 \lambda$, respectively. We put 20 cells per wavelength in each direction. The left and right vacuum occupy $35 \lambda$ and $45 \lambda$, respectively, and the rest is occupied by plasma. In the front of the plasma, a $10 \lambda$ linear density ramp is added. The homogeneous plasma density is $n_{e}=0.08$, and the inertial electron temperature is $T_{e}=100 \mathrm{eV}$. The semi-infinite pump laser is s-polarized with the uniform amplitude $a_{0}=0.05$, with a $25 \lambda$ rising edge in the front. The radius of the focal spot is $10 \lambda$, and the center of it is located at $y=30 \lambda$. The transverse intensity profile of laser satisfies Gaussian distribution. The ion mass is $m_{i}=1836 m_{e}$.

Figure 5(a) describes the early stage of side scattering without bandwidth. Later at $t=500 \tau$ the intensity of the scat-
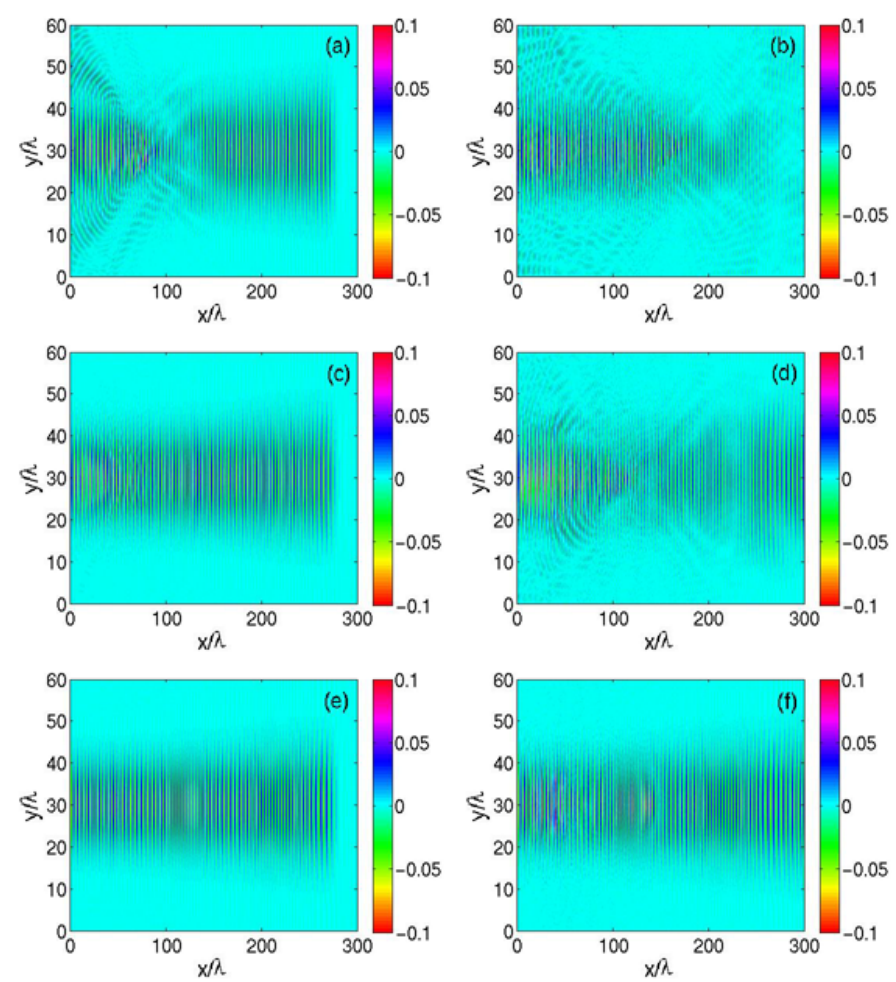

FIG. 5. Comparison of electric fields with or without finite bandwidths. (a) and (b) are the $E_{z}$ distributions without bandwidth at $t=300 \tau$ and $t=500 \tau$, respectively. (c) and (d) are the $E_{z}$ distributions with $5 \%$ bandwidth at $t=300 \tau$ and $t=500 \tau$, respectively. (e) and (f) are the $E_{z}$ distributions with $10 \%$ bandwidth at $t=300 \tau$ and $t=500 \tau$, respectively. The normalized amplitude of the incident laser is $a_{0}=0.05$ and the plasma density is $n_{e}=0.08$.

tering light becomes stronger, as shown in Fig. 5(b). When $\triangle \omega=2 \%$, its effect is not obvious and the distribution of $E_{z}$ is almost the same as the one with $\triangle \omega=0$. When the bandwidth is increased to 5\%, the SRS suppression becomes obvious as shown in Fig 5(c) at $t=300 \tau$. One may note that at $t=500 \tau$ there is some side scattering, but its intensity is still lower than the one with $\triangle \omega=0$, as shown in Fig. 5(d). When taking the bandwidth $\triangle \omega=10 \%$, side scattering is suppressed more significantly. We cannot see obviously side scattering in Fig. 5(e) and (f). To study this in details, we compare the spectra of the longitudinal electric field $E_{x}$ in $k$ space, as shown in Fig. 6. One may note that the strongest region are around $k=1.62$ which is the same as in the $1 \mathrm{D}$ case. With $10 \%$ bandwidth, the divergence angle of $E_{x}$ is much smaller than the one without bandwidth, indicating $10 \%$ bandwidth can suppress the side scattering significantly. However, as shown in Fig. 6(b), the side scattering does not disappear even using a relatively large bandwidth.

Figure 7 gives the temporal evolution and the spectra of the backscattering light with or without finite bandwidths. The diagnosis position is located at $x=5 \lambda$ and $y=30 \lambda$. The results are quite similar to $1 \mathrm{D}$ simulations given in Sec. IIB. With finite bandwidths, for example, $\triangle \omega=5 \%$, the amplitude of the backscattering light grows more slowly compared 

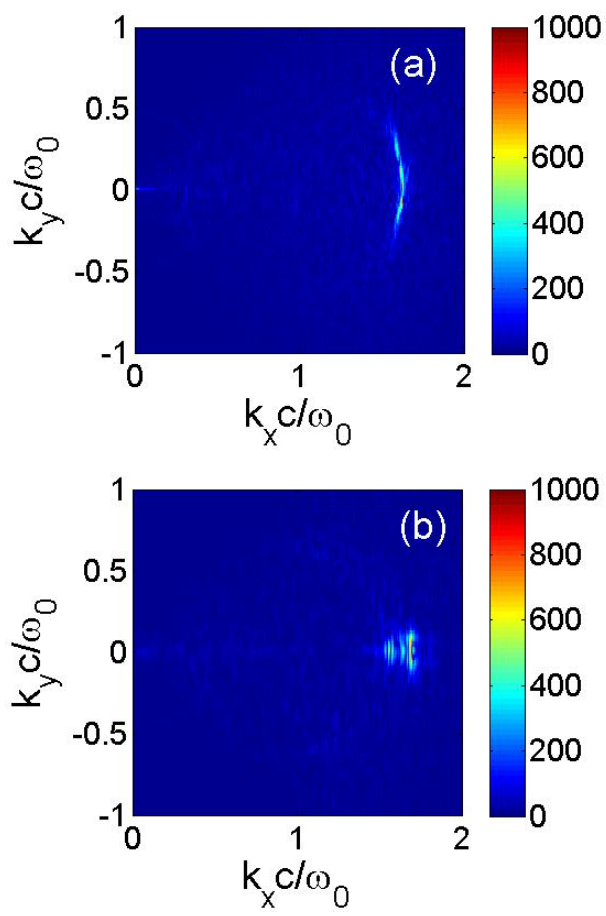

FIG. 6. The distribution of the longitudinal electric field $E_{x}$ in the $k$ space. (a) Without laser bandwidth, at $t=500 \tau$. (b) With $\triangle \omega=$ $10 \%$, at $t=500 \tau$. The normalized amplitude of the incident laser is $a_{0}=0.05$ and the plasma density is $n_{e}=0.08$.

to the one without bandwidth. Meanwhile, the intensity of the spectrum is lower. The larger the bandwidth, the stronger the suppression effects. Compared with Fig. 2(b), it shows that the scattered light near $\omega / \omega_{0}=1$ due to the stimulated Brillouin scattering (SBS) is much weak in Fig. 7(b). This is because the simulation time in the 2D simulation is limited up to $500 \tau$, much shorter than that in the 1D simulation.

Since SRS instability produces hot electrons through electron plasma wave excitations, therefore the level of hot electron generation is also a useful measure of the SRS development. From Fig. 8(a) one can see that at $t=300 \tau$, in the front of the plasma, the maximum normalized longitudinal momentum of the electrons $p_{x}$ is close to 0.6 without introducing bandwidth. In the case with $\triangle \omega=10 \%$, the maximum $p_{x}$ is much reduced. However, at later $t=500 \tau, p_{x}$ in the front of the plasma have similar values as the ones without bandwidth, as shown in Fig. 8(b). This means the bandwidth only delays the linear growth time of the SRS, but it cannot completely suppress the SRS. These results are very similar to the $1 \mathrm{D}$ cases. Since side scattering can drive transverse electron plasma waves, they can heat electrons in the transverse direction. From Fig. 8(c) we can see that at $t=300 \tau$, the transverse momentum $p_{y}$ of the electrons are relatively small with $\triangle \omega=10 \%$. At $t=500 \tau, p_{y}$ in the front of the plasma grow to the same level as the case without finite bandwidth, which also means the bandwidth can only reduce the side scattering level at the beginning, but cannot eliminate it.

Generally the energy spectra of the hot electrons can reflect
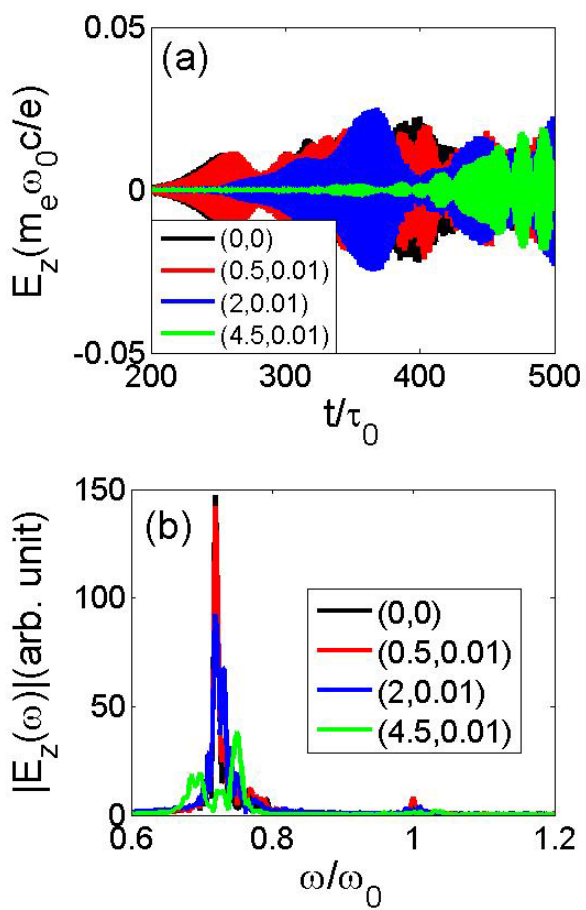

FIG. 7. (a) Temporal evolution of the backscattering light with or without finite bandwidth at $x=5 \lambda$ and $y=30 \lambda$. (b) Fourier transform of the backscattering light in (a). The normalized amplitude of the incident laser is $a_{0}=0.05$, and the plasma density is $n_{e}=0.08$.

the level of the SRS. Figure 9 shows the electron energy distributions at different times under the same laser and plasma conditions as in Fig. 8. At $t=300 \tau$, the spectra for $\triangle \omega=0$ and $\triangle \omega=2 \%$ are almost the same, while the spectrum for $\triangle \omega=5 \%$ is much lower than them. When the bandwidth up to $10 \%$, the electron energies are reduced significantly. This agrees with the previous observation with the scattered light that the larger the bandwidth, the stronger the suppression effects of the SRS. However, with the increase of the laserplasma interaction time and the nonlinear development of the SRS, the hot electron temperatures for $\triangle \omega=5 \%$ and $10 \%$ increase so much that their spectra are approaching to those found for $\triangle \omega=0,2 \%$ at $t=500 \tau$.

\section{B. Effects of other laser and plasma parameters}

Besides the bandwidth itself, other laser and plasma parameters can also affect the suppression effects on the SRS. Analytical theory only gives the relation between the linear growth rate and bandwidth magnitude. Via PIC simulations, we find that for the same bandwidth, the suppression effects will be different for the different frequency modulation parameter $f_{m}$. It is found that there is a proper range for $f_{m}$ to have the strongest suppression effects on the SRS. For a constant $\triangle \omega$, a large $f_{m}$ leads to a small number of peaks in spectrum. Also if $f_{m}$ is too small, the discrete spectrum becomes approximately continuous. Both of them are not advantageous 

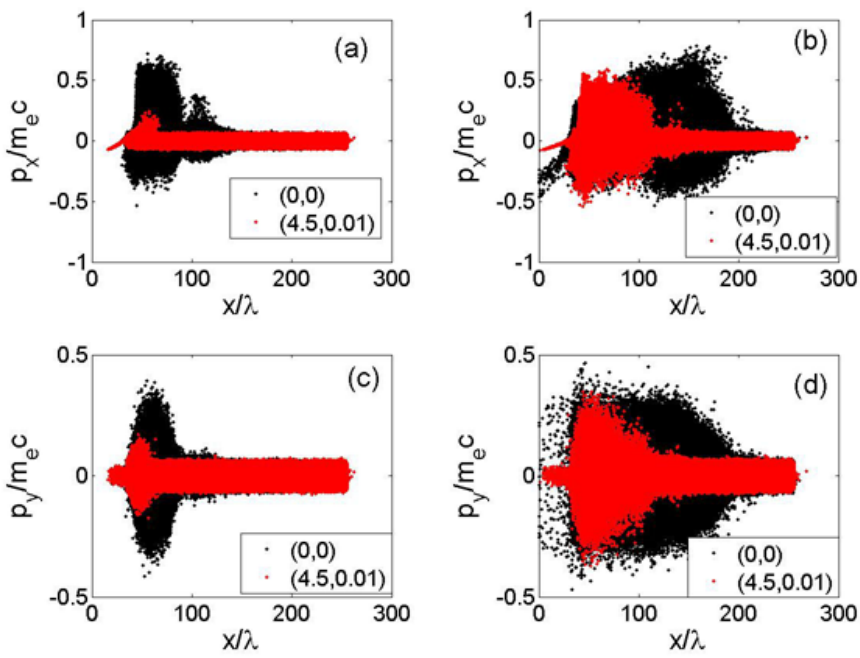

FIG. 8. (a) and (b) are the longitudinal momentum of the electrons at $t=300 \tau$ and $t=500 \tau$, respectively. (c) and (d) are the transverse momentum of the electrons at $t=300 \tau$ and $t=500 \tau$, respectively. The normalized amplitude of the incident laser is $a_{0}=0.05$, and the plasma density is $n_{e}=0.08$.

to bandwidth suppression effects. Here we fix the bandwidth $\triangle \omega=10 \%$, and change $f_{m}=0.002,0.005,0.01$. Again, we use the hot electron energy spectrum as a measure of the SRS. The comparison of the electron energy spectra are shown in Fig. 10(a). It shows that at $t=500 \tau$, the energy spectrum for $f_{m}=0.005$ is the lowest, which indicates the best suppression effects on SRS. This calculation indicates that even with the fixed bandwidth, different modulation parameter $f_{m}$ can lead to different suppression effects.

As mentioned above, when the incident laser has a bandwidth $\triangle \omega$, the growth rate of the SRS becomes $\Gamma_{0}^{2} / \triangle$ $\omega$. Therefore, besides increasing the bandwidth, one can see stronger suppression effects of the finite bandwidth by decreasing the linear growth rate $\Gamma_{0}$. The maximum growth rate of stimulated Raman backscattering is $\Gamma_{0}=$ $\sqrt{3}\left(\omega_{0} \omega_{p}^{2} a_{0}^{2} / 16\right)^{1 / 3} \cdot{ }^{18}$ By decreasing the amplitude of the incident laser or the plasma density, one can reduce $\Gamma_{0}$. As shown in Fig. 10(b), when the plasma density is reduced to $n_{e}=0.03$, the reduction of the produced hot electron temperature is more obviously, indicating the suppression effects of the bandwidth on the SRS is stronger. When the amplitude of incident laser is increased, the suppression effects of laser bandwidth become weaker. As an example, Fig. 10(c) shows that when the amplitude is enhanced to $a_{0}=0.08$, the bandwidth suppression effects appear not significant at all.

Analytical theory is usually given based on the cold plasma model, but we find that the initial plasma electron temperature has a significant impact on the bandwidth suppression effects. As shown in Fig. 10(d) and (e), when $T_{e}=1 \mathrm{keV}$, the SRS can be suppressed considerably with a $10 \%$ bandwidth. While with the increase of the plasma temperature up to $5 \mathrm{keV}$, the bandwidth suppression effects become weaker. For the laser plasma parameters under consideration, we found that when the electron temperature is higher than $5 \mathrm{keV}$, the bandwidth
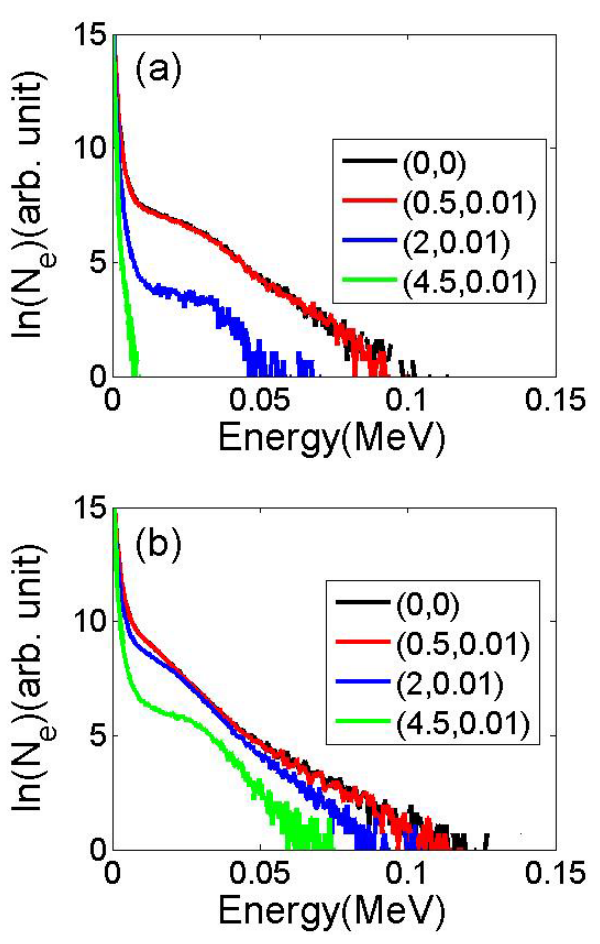

FIG. 9. (a) and (b) are the electron energy spectra with different bandwidth parameters $\left(b, f_{m}\right)$ at $t=300 \tau$ and $t=500 \tau$, respectively. The normalized amplitude of the incident laser is $a_{0}=0.05$ and the plasma density is $n_{e}=0.08$.

suppression effects become very weak. At this temperature, the linear growth rate of the SRS does not yet change considerably by the electron temperature ${ }^{26}$. However, at high electron temperatures, the fluctuation fields in plasma become higher. These fluctuations and large-number electrons near the phase velocity of plasma wave allow the SRS instability to grow to the nonlinear stage in a shorter time, which cannot be suppressed by the laser bandwidth. So the bandwidth effects become weaker at high temperatures as shown above. In passing, in order to avoid possible overestimation of fluctuations in PIC simulations due to numerical noise, one needs to adopt high resolutions and more particles per cell for this problem.

\section{SUMMARY}

The effects of the laser bandwidth on the SRS instabilities have been investigated via PIC simulations. For the laser pulse with sinusoidal frequency modulation under consideration, both one and two dimensional PIC simulations indicate that SRS can be suppressed significantly when the laser bandwidth is much larger than the linear growth rate, in agreement with the theory. In order to have strong suppression effects, the laser bandwidth should be larger than 5\% under the conditions of inertial confinement fusion. The bandwidth can also suppress stimulated Raman side scattering when the size of the bandwidth is larger than 5\%. On the other hand, simulation results suggest that the bandwidth only reduces the linear 

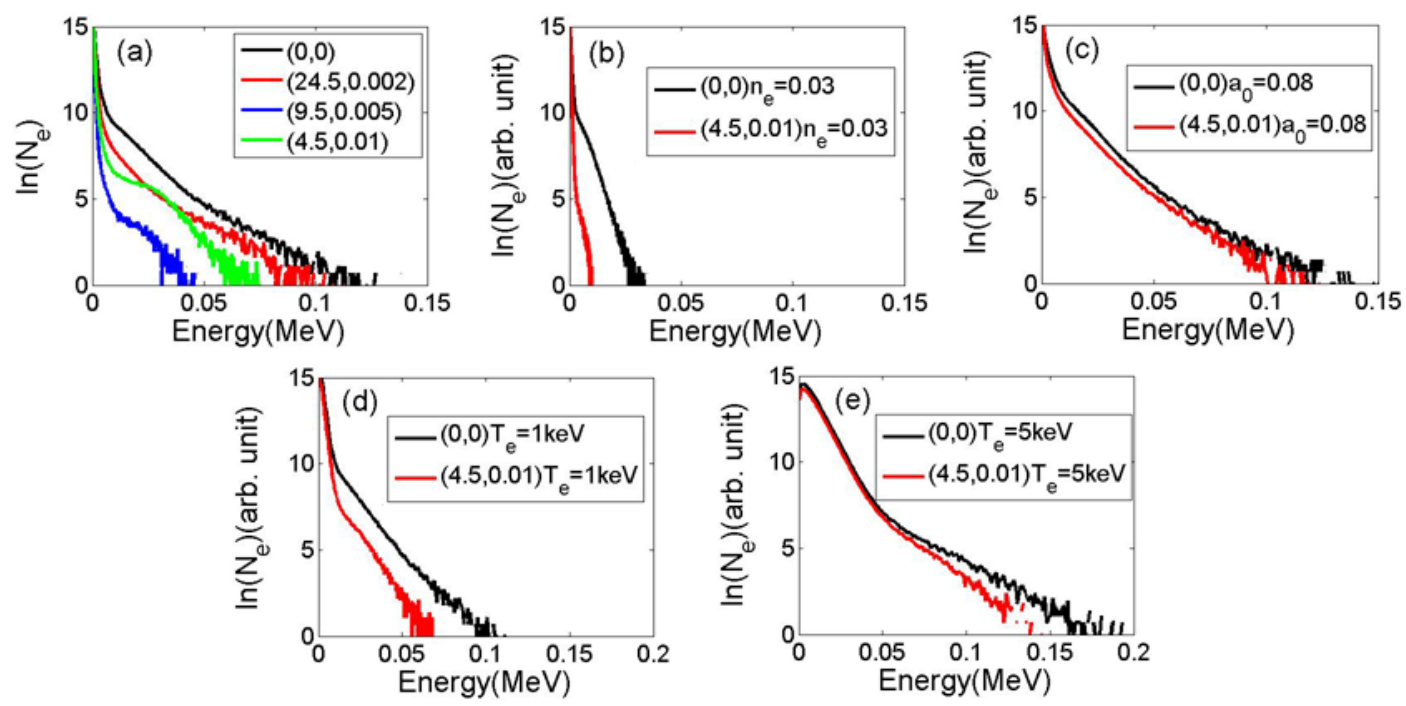

FIG. 10. Electron energy spectra with different laser and plasma parameters at $t=500 \tau$. (a) With different frequency modulation parameters $\left(b, f_{m}\right)$, where the normalized amplitude $a_{0}=0.05$, and the plasma density $n_{e}=0.08$. (b) With laser amplitude is $a_{0}=0.05$ and plasma density $n_{e}=0.03$. (c) With laser amplitude $a_{0}=0.08$ and plasma density is $n_{e}=0.08$. (d) and (e) are the electron energy spectra with different plasma temperatures at $T_{e}=1 \mathrm{keV}$ and $5 \mathrm{keV}$, respectively, where the laser amplitude $a_{0}=0.05$ and the plasma density $n_{e}=0.08$.

growth rate of the SRS. With the increase of the laser-plasma interaction time, the SRS still can develop to a high level comparable to that found without finite laser bandwidth.

Besides the bandwidth magnitude, the frequency modulation parameter $f_{m}$ can also affect the suppression effects. It has an optimal range for the suppression effects, i.e., if $f_{m}$ is either too large or too small, the suppression effects will get weaker or disappear. It is also found that by decreasing the amplitude of laser or the plasma density, the suppression effects can be made more significant by reducing the linear growth rate of the SRS. Initial plasma electron temperature can weaken the bandwidth effects. For the laser plasma parameters under consideration, when the plasma temperature is larger than $5 \mathrm{keV}$, laser bandwidth effects almost disappear. It should be pointed out that the bandwidth effects shown in this work is based upon the particular model of laser fields. For a given bandwidth but with different temporal structures of the laser fields, the bandwidth effects need to be checked separately.

\section{ACKNOWLEDGMENTS}

This work was supported in part by the National Basic Research Program of China (Grant No. 2013CBA01504), the National Science Foundation of China (Grant Nos. 11421064, 11129503, 11374209, 11405107 and 11374210), the MOST international collaboration project (Grant No. 2014DFG02330). C.R. is also supported by U.S. DOE under Grant Nos. DE-FC02-04ER54789 and DE-SC0012316.

${ }^{1}$ W. L. Kruer et al., The physics of laser plasma interactions, Vol. 70 (Addison-Wesley New York, 1988).

${ }^{2}$ J. C. Fernández, J. Cobble, D. Montgomery, M. Wilke, and B. Afeyan, Phys. Plasmas 7, 3743 (2000).
${ }^{3}$ D. Umstadter, S.-Y. Chen, R. Wagner, A. Maksimchuk, and G. Sarkisov, Opt. Express 2, 282 (1998).

${ }^{4}$ A. Modena, Z. Najmudin, A. Dangor, C. Clayton, K. Marsh, C. Joshi, V. Malka, C. Darrow, C. Danson, D. Neely, et al., Nature 377, 606 (1995).

${ }^{5}$ J. Workman, A. Maksimchuk, X. Liu, U. Ellenberger, J. Coe, C.-Y. Chien, and D. Umstadter, JOSA B 13, 125 (1996).

${ }^{6}$ E. S. Dodd and D. Umstadter, Phys. Plasmas 8, 3531 (2001).

${ }^{7}$ S. Glenzer, P. Arnold, G. Bardsley, R. Berger, G. Bonanno, T. Borger, D. Bower, M. Bowers, R. Bryant, S. Buckman, et al., Nucl. Fusion 44, S185 (2004).

${ }^{8}$ J. Lindl, Phys. Plasmas 2, 3933 (1995).

${ }^{9}$ R. Drake, R. Turner, B. Lasinski, K. Estabrook, E. Campbell, C. Wang, D. Phillion, E. Williams, and W. Kruer, Phys. Rev. Lett. 53, 1739 (1984).

${ }^{10}$ S. Glenzer, B. MacGowan, P. Michel, N. Meezan, L. Suter, S. Dixit, J. Kline, G. Kyrala, D. Bradley, D. Callahan, et al., Science 327, 1228 (2010).

${ }^{11}$ E. Williams, J. Albritton, and M. Rosenbluth, Phys. Fluids 22, 139 (1979).

${ }^{12}$ J. J. Thomson and J. I. Karush, Phys. Fluids 17, 1608 (1974).

${ }^{13}$ B. Afeyan and S. Hüller, in EPJ Web of Conferences, Vol. 59 (EDP Sciences, 2013) p. 05009.

${ }^{14}$ S. Obenschain, N. Luhmann Jr, and P. Greiling, Phys. Rev. Lett. 36, 1309 (1976).

${ }^{15}$ P. Guzdar, C. Liu, and R. Lehmberg, Phys. Fluids B 3, 2882 (1991).

${ }^{16}$ D. Herrmann, C. Homann, R. Tautz, M. Scharrer, P. S. J. Russell, F. Krausz,

L. Veisz, and E. Riedle, Opt. Express 18, 18752 (2010).

${ }^{17}$ D. Herrmann, L. Veisz, R. Tautz, F. Tavella, K. Schmid, V. Pervak, and F. Krausz, Opt. Letters 34, 2459 (2009).

${ }^{18}$ D. Forslund, J. Kindel, and E. Lindman, Phys. Fluids 18, 1002 (1975).

${ }^{19}$ M. Chen, Z.-M. Sheng, J. Zheng, Y.-Y. Ma, and J. Zhang, Chin. J. Comput. Phys. 25, 50 (2008).

${ }^{20}$ S. Hüller, P. Mounaix, and V. Tikhonchuk, Phys. Plasmas 5, 2706 (1998).

${ }^{21}$ R. Berger, B. Lasinski, A. Langdon, T. Kaiser, B. Afeyan, B. Cohen, C. Stil1, and E. Williams, Phys. Rev. Lett. 75, 1078 (1995).

${ }^{22}$ Y. Zhao, J. Zheng, L.-L. Yu, M. Chen, S.-M. Weng, and Z.-M. Sheng, Sci China G-Phys Mech Astron 45, 035201 (2015).

${ }^{23}$ K. Mima, M. Jovanović, Y. Sentoku, Z.-M. Sheng, M. Škorić, and T. Sato, Phys. Plasmas 8, 2349 (2001).

${ }^{24}$ B. Winjum, J. Fahlen, F. Tsung, and W. Mori, Phys. Rev. Lett. 110, 165001 (2013).

${ }^{25}$ H. Jang, M. Hur, J. Lee, M. Cho, W. Namkung, and H. Suk, Appl. Phys. Lett. 93, 071506 (2008).

${ }^{26}$ Y. Zhao, J. Zheng, M. Chen, L.-L. Yu, S.-M. Weng, C. Ren, C.-S. Liu, and 
Z.-M. Sheng, Phys. Plasmas 21, 112114 (2014).

${ }^{27}$ H. A. Rose and D. A. Russell, Phys. Plasmas 8, 4784 (2001).

${ }^{28}$ W. Rozmus, R. Sharma, J. Samson, and W. Tighe, Phys. Fluids 30, 2181 (1987).

${ }^{29}$ G. Bonnaud, D. Pesme, and R. Pellat, Phys. Fluids B 2, 1618 (1990).

${ }^{30}$ L. Nikolić, M. Škorić, S. Ishiguro, and T. Sato, Phys. Rev. E 66, 036404 (2002).

${ }^{1}$ W. L. Kruer et al., The physics of laser plasma interactions, Vol. 70 (Addison-Wesley New York, 1988).

${ }^{2}$ J. C. Fernández, J. Cobble, D. Montgomery, M. Wilke, and B. Afeyan, Phys. Plasmas 7, 3743 (2000).

${ }^{3}$ D. Umstadter, S.-Y. Chen, R. Wagner, A. Maksimchuk, and G. Sarkisov, Opt. Express 2, 282 (1998).

${ }^{4}$ A. Modena, Z. Najmudin, A. Dangor, C. Clayton, K. Marsh, C. Joshi,

V. Malka, C. Darrow, C. Danson, D. Neely, et al., Nature 377, 606 (1995).

${ }^{5}$ J. Workman, A. Maksimchuk, X. Liu, U. Ellenberger, J. Coe, C.-Y. Chien, and D. Umstadter, JOSA B 13, 125 (1996).

${ }^{6}$ E. S. Dodd and D. Umstadter, Phys. Plasmas 8, 3531 (2001).

${ }^{7}$ S. Glenzer, P. Arnold, G. Bardsley, R. Berger, G. Bonanno, T. Borger, D. Bower, M. Bowers, R. Bryant, S. Buckman, et al., Nucl. Fusion 44, S185 (2004).

${ }^{8}$ J. Lindl, Phys. Plasmas 2, 3933 (1995).

${ }^{9}$ R. Drake, R. Turner, B. Lasinski, K. Estabrook, E. Campbell, C. Wang,

D. Phillion, E. Williams, and W. Kruer, Phys. Rev. Lett. 53, 1739 (1984).

${ }^{10}$ S. Glenzer, B. MacGowan, P. Michel, N. Meezan, L. Suter, S. Dixit, J. Kline, G. Kyrala, D. Bradley, D. Callahan, et al., Science 327, 1228 (2010).

${ }^{11}$ E. Williams, J. Albritton, and M. Rosenbluth, Phys. Fluids 22, 139 (1979).

${ }^{12}$ J. J. Thomson and J. I. Karush, Phys. Fluids 17, 1608 (1974).
${ }^{13}$ B. Afeyan and S. Hüller, in EPJ Web of Conferences, Vol. 59 (EDP Sciences, 2013) p. 05009

${ }^{14}$ S. Obenschain, N. Luhmann Jr, and P. Greiling, Phys. Rev. Lett. 36, 1309 (1976).

${ }^{15}$ P. Guzdar, C. Liu, and R. Lehmberg, Phys. Fluids B 3, 2882 (1991).

${ }^{16}$ D. Herrmann, C. Homann, R. Tautz, M. Scharrer, P. S. J. Russell, F. Krausz, L. Veisz, and E. Riedle, Opt. Express 18, 18752 (2010).

${ }^{17}$ D. Herrmann, L. Veisz, R. Tautz, F. Tavella, K. Schmid, V. Pervak, and F. Krausz, Opt. Letters 34, 2459 (2009).

${ }^{18}$ D. Forslund, J. Kindel, and E. Lindman, Phys. Fluids 18, 1002 (1975).

${ }^{19}$ M. Chen, Z.-M. Sheng, J. Zheng, Y.-Y. Ma, and J. Zhang, Chin. J. Comput. Phys. 25, 50 (2008).

${ }^{20}$ S. Hüller, P. Mounaix, and V. Tikhonchuk, Phys. Plasmas 5, 2706 (1998).

${ }^{21}$ R. Berger, B. Lasinski, A. Langdon, T. Kaiser, B. Afeyan, B. Cohen, C. Stil1, and E. Williams, Phys. Rev. Lett. 75, 1078 (1995).

${ }^{22}$ Y. Zhao, J. Zheng, L.-L. Yu, M. Chen, S.-M. Weng, and Z.-M. Sheng, Sci China G-Phys Mech Astron 45, 035201 (2015).

${ }^{23}$ K. Mima, M. Jovanović, Y. Sentoku, Z.-M. Sheng, M. Škorić, and T. Sato, Phys. Plasmas 8, 2349 (2001).

${ }^{24}$ B. Winjum, J. Fahlen, F. Tsung, and W. Mori, Phys. Rev. Lett. 110, 165001 (2013).

${ }^{25}$ H. Jang, M. Hur, J. Lee, M. Cho, W. Namkung, and H. Suk, Appl. Phys. Lett. 93, 071506 (2008).

${ }^{26}$ Y. Zhao, J. Zheng, M. Chen, L.-L. Yu, S.-M. Weng, C. Ren, C.-S. Liu, and Z.-M. Sheng, Phys. Plasmas 21, 112114 (2014).

${ }^{27}$ H. A. Rose and D. A. Russell, Phys. Plasmas 8, 4784 (2001).

${ }^{28}$ W. Rozmus, R. Sharma, J. Samson, and W. Tighe, Phys. Fluids 30, 2181 (1987).

${ }^{29}$ G. Bonnaud, D. Pesme, and R. Pellat, Phys. Fluids B 2, 1618 (1990).

${ }^{30}$ L. Nikolić, M. Škorić, S. Ishiguro, and T. Sato, Phys. Rev. E 66, 036404 (2002). 